The Junior Trainees Group of BAOMS updates this information annually and puts it on the BAOMS website.

Also BAOMS advertises every year in this journal as well as the British Medical Journal and NHS jobs for people to register their interest in oral and maxillofacial surgery. All who register, using the link on the BAOMS website, are sent information about training in OMFS including applying to medical school.

Most disappointing, they did not mention two events which happen every year. Firstly the BAOMS National Training and Careers Day (NTCD) for those interested in OMFS and OS. This has five streams of talks and small group tutorials aimed at those considering applying for their second degree, trainees studying for their second degree, those dually qualified in foundation/core training aiming to enter OMFS at ST3, a research stream and, new this year, an oral surgery stream. The cost of the day is nominal, and it runs parallel to the specialist registrars conference (who are also a valuable source of information). The day is book-ended by lectures from two key-note speakers. It is timed to happen before UCAS forms need to be submitted. In 2010 it will be in York on Friday 1 October. Details are on the BAOMS website and trainees are encouraged to ask for information that they need to be included http://www.baoms.org.uk/ page.asp?id=521. Secondly, The Junior Trainees Group Annual Conference always has a careers advice section.

Finally, and very importantly, the authors did not mention the value of working in an SHO post in a unit with close links to their medical school.

We are sure that the admissions tutors are busy people, and might become vexed when asked the same information by a number of individuals, and would encourage the authors to work with the Junior Trainees Group of BAOMS if they plan to update this information for next year. We know it is hard work collecting the information. We did it for ten years!

P. Magennis, T. K. Ong By email

1. Pepper T, Tabiat-Pour S. Getting into medical school - a guide for dental graduates. Br Dent J 2010; 208: 123-126.

\section{MEDICAL SCHOOL SUPPORT}

Sir, we read with great delight and some disappointment the article Getting into medical school - a guide for dental graduates. ${ }^{1}$ We are extremely happy to see such an article published, however, disappointed that the author felt that there was little information available prior to their application to medical school as a dental graduate. We would like to highlight the additional sources of information that will benefit all applicants.

The primary source of information and guidance comes from The Junior Trainees' Group of the British Association of Oral and Maxillofacial Surgeons (JTG of BAOMS) and the annual National Training Day (NTD). The JTG of BAOMS delivers application information via the group's online forum, the trainees' section of the BAOMS website, and vitally through the annual JTG of BAOMS conference which has a session solely on education and training. Further to this the NTD has a dedicated 'applying to medical school stream', which provides seminars on the application process delivered by individuals who have recently been through it.

The JTG of BAOMS conference, NTD and online forum offers potential applicants a chance to meet individuals in the same position and seek advice from those who have successfully applied to medical school all in a relaxed and nonthreatening environment.

These lines of support are further backed up by adverts in the $B D J$ and $B M J$ to register your interest in oral and maxillofacial surgery on the BAOMS website. By registering your interest you are automatically added to a mailing list from which you receive all the current and relevant information to your stage of training. This is an invaluable source of information to all trainees.

We hope this provides extra guidance and support for any dentist applying to medical school and thank the authors for their article.

N. Scott, E. Gruber By email

1. Pepper T, Tabiat-Pour S. Getting into medical school - a guide for dental graduates. Br Dent J 2010; 208: 123-126.

DOI: $10.1038 /$ sj.bdj.2010.303 